\title{
Changes in Fluorescence, Color, and Morphology of Fluorescent Nanostructures under Successive Light Irradiation
}

\author{
Khin Moe Lwin ${ }^{1,2}$, Sanghyuk Park ${ }^{1^{*}}$, and Mina Han ${ }^{2 * *}$ \\ ${ }^{1}$ Department of Chemistry, \\ ${ }^{2}$ Department of Chemistry Education, \\ Kongju National University, 56 Gongjudaehak-ro, Gongju, Chungnam 32588, Korea \\ *spark0920@kongju.ac.kr \\ **hanmin@kongju.ac.kr
}

\begin{abstract}
Fluorescence, color, and morphological changes of fluorescent nanostructures consisting of azobenzene-containing chromophores were investigated under successive visible light irradiation. Compared with spheres composed of a chromophore showing sufficient photoisomerization, fluorescent nanofibers assembled from a molecule showing a very small amount of photoisomerization had a slow response to light. Successive visible light irradiation of the nanofibers led to changes in fluorescence intensity and color, whereas the one-dimensional morphology was hardly affected. UV-visible absorption spectroscopy, optical microscopy (OM), scanning electron microscopy (SEM), and fluorescence optical microscopy (FOM) were employed to confirm the light-responsive properties. Our observations suggest that sufficient photoisomerization plays a key role in realizing fast and efficient light response of self-assembled structures.
\end{abstract}

Keywords: Color, Fluorescent Nanostructure, Light, Morphology

\section{Introduction}

Stimuli-responsive fluorescent materials have received considerable attention owing to their fundamental researches and practical applications in sensors, actuators, display, and memory devices [15]. To alter the morphology and fluorescence property of self-assembled nanostructures, supramolecular systems are formed through noncovalent interactions including $\pi-\pi$ stacking, van der Waals, hydrophobic effects, electrostatic interactions, and so on [6-11]. Fluorescent organic materials that respond to external stimuli such as light, temperature, mechanical forces and chemicals have been developed [1-7,12]. Compared with other stimuli, light acts as a useful trigger because it is possible to tune its wavelength and intensity a noncontact and remote-controlled manner.

Han et al. designed a new type of trigonal azobenzene-containing chromophore $(3 \mathrm{BrAz}$ and $3 \mathrm{BuAz}$, see Scheme 1) with aggregation-induced emission/aggregation-induced

emission enhancement (AIE/AIEE) property [3,4]. 3BuAz underwent sufficient photoisomerization $(\sim 90 \%)$ and the self-assembled spheres exhibited significant light-driven morphological changes and the resulting decreases in fluorescence intensity [13]. However, although red fluorescence from $3 \mathrm{BrAz}$ nanofibers was reduced by external stimuli such as pressure, friction, and thermal treatment owing to decreases in the degree of crystallinity [14], the light-responsiveness has not been explored yet on the basis of the light-induced molecular structure
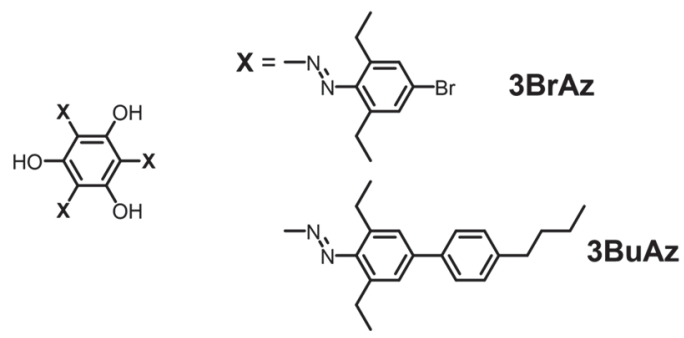

Scheme 1. 
change. Here, we investigated the photoisomerization behavior of $3 \mathrm{BrAz}$ in dilute solution and the light response of $3 \mathrm{BrAz}$ nanofibers. In contrast to $3 \mathrm{BuAz}$, the molecular structure of $3 \mathrm{BrAz}$ was rarely changed by light even in solution. Changes in fluorescence, color and morphology of the $3 \mathrm{BrAz}$ nanofibers by successive light irradiation were monitored using optical microscopy (OM), fluorescence optical microscopy (FOM), and scanning electron microscopy (SEM) experiments.

\section{Experimental}

Spectroscopic-grade tetrahydrofuran (THF) solvent was used to dissolve $3 \mathrm{BrAz}$ and $3 \mathrm{BuAz}$. Each azobenzene solution was placed in a clean quartz cell and sealed with parafilm after 30 seconds nitrogen purging. The absorption and fluorescence spectra were taken after 30 and 40 min of sample preparation, respectively.

To produce self-assembled structures, we added water dropwise to the $3 \mathrm{BrAz}$ in THF solution with gentle shaking. As the water content increased, the mixture solution started turning turbid and a floating suspension was produced. Largely entangled fiber wads separated slowly from the solvent mixture. OM, FOM and SEM samples were then prepared by placing one drop of the red entangled fiber wad on a clean glass substrate and allowing it to fully dry at room temperature.

UV-vis absorption and fluorescence spectra were obtained using a Shimadzu UV-2600 UV-vis spectrophotometer and a Horiba FluoroMax-4 spectrofluorometer, respectively. The SEM samples were first coated with a platinum layer for 60 seconds using a Hitachi E-1030 ion sputter. The SEM images were recorded by using a TESKANMIRA3-LM microscope. The optical microscopy and fluorescence optical microscopy images were obtained using an Olympus BX53 microscope equipped with an Olympus WIG fluorescence cube $\left(\lambda_{\mathrm{ex}}=520-550 \mathrm{~nm}\right)$.

\section{Results and discussion}

UV-visible absorption spectroscopy was used to monitor the light-induced molecular structure change of $3 \mathrm{BrAz}$ in dilute THF solution. The absorption spectrum of freshly prepared $3 \mathrm{BrAz}$ solution displayed two strong absorption bands centered at 362 and $478 \mathrm{~nm}$, and red-shifted absorption bands were recorded in the $3 \mathrm{BuAz}$ spectrum (Fig. 1a). $3 \mathrm{BrAz}$ and $3 \mathrm{BuAz}$ solutions showed very weak fluorescence centered at around 570 and $610 \mathrm{~nm}$, respectively (Fig. 1b). Figure 1c
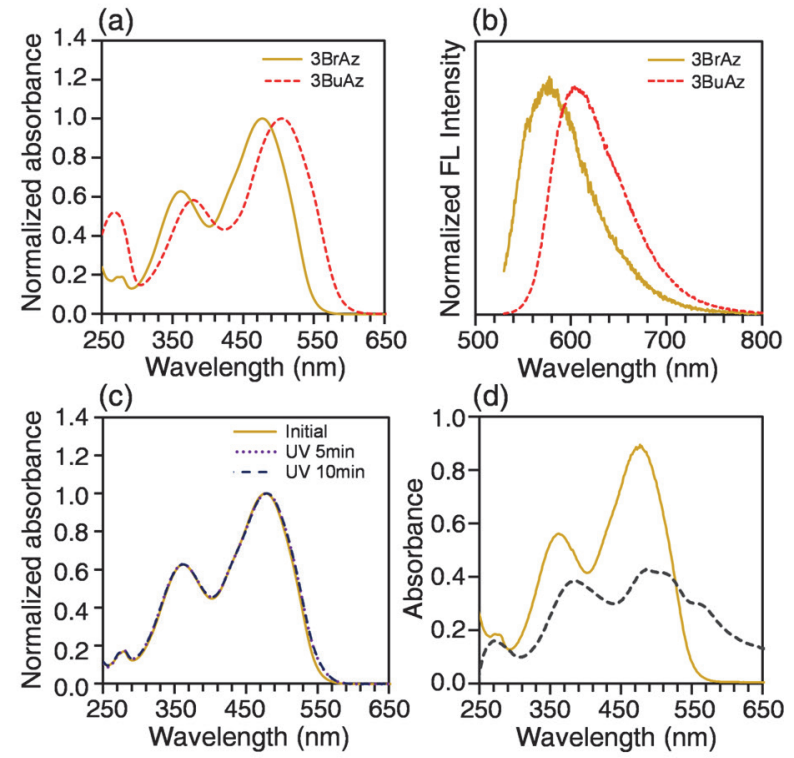

Fig. 1. (a) UV-vis absorption spectra of $3 \mathrm{BrAz}$ and $3 \mathrm{BuAz}$ in THF. (b) Fluorescence spectra of $3 \mathrm{BrAz}$ and $3 \mathrm{BuAz}$ in THF $\left(\lambda_{\mathrm{ex}}=510 \mathrm{~nm}\right)$. (c) Absorption spectral changes of $3 \mathrm{BrAz}$ in THF as a function of exposure time of UV light at $365 \mathrm{~nm}$. (d) Absorption spectra before (solid line) and after (dashed line) the formation of $3 \mathrm{BrAz}$ fibers.

shows UV-visible absorption spectral changes of $3 \mathrm{BrAz}$ solution as a function of exposure time of UV light at $365 \mathrm{~nm}$. Even after irradiation with UV light for $10 \mathrm{~min}$, two characteristic absorption bands were changed by only $<4 \%$. That is, light irradiation scarcely seemed to generate an isomer with a sufficient lifetime $[15,16]$. This is in sharp contrast to $3 \mathrm{BuAz}$ which showed $\sim 90 \%$ of light-induced molecular structure changes and slow thermal back isomerization [13].

Self-assembled structures were formed using a simple reprecipitation method. As water was added into $3 \mathrm{BrAz}$ THF solution, the mixture solution started turning turbid due to the occurrence of selfassembled aggregates. Subsequently, largely entangled $3 \mathrm{BrAz}$ fiber wads slowly sank to the bottom of the quartz cell. Upon the formation of fibers, two characteristic absorption bands were redshifted and a shoulder band at around $560 \mathrm{~nm}$ appeared (Fig. 1d). These spectral changes would be associated with inclined molecular arrangements of the chromophores in the fibers (i.e., J-aggregation).

$\mathrm{OM}$ and SEM measurements revealed that reddish-brown $3 \mathrm{BrAz}$ fibers were $<100 \mathrm{~nm}$ wide and over tens of micrometers long (Figs. 2a and 3b). This observation is in agreement with the previous study [14]. The narrow nanofibers tended to 

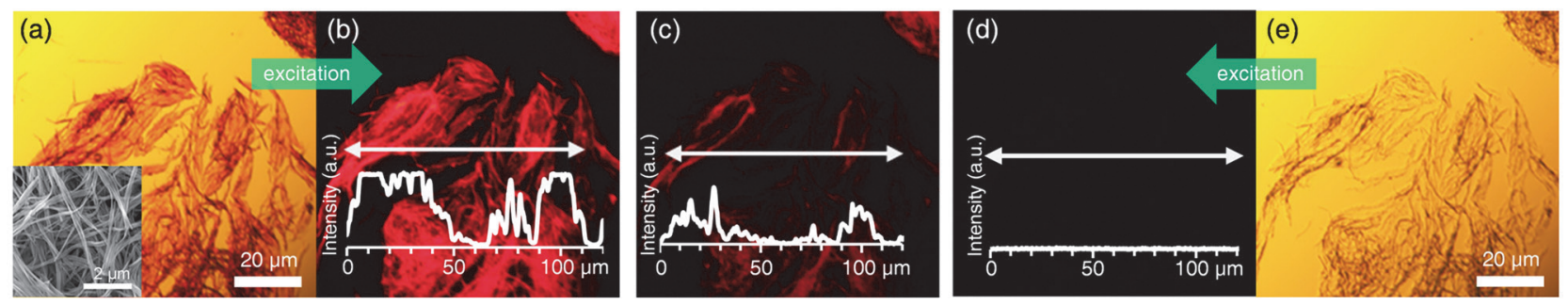

Fig. 2 (a) OM, SEM (inset), and (b) FOM $\left(\lambda_{\mathrm{ex}}=520-550 \mathrm{~nm}\right.$ ) images of $3 \mathrm{BrAz}$ nanofibers prepared from $5 \times 10^{-5} \mathrm{M}$ $\mathrm{THF} / \mathrm{H}_{2} \mathrm{O}(1: 4, \mathrm{v} / \mathrm{v})$. (c) FOM image of nanofibers taken after irradiation with green light for 6 min. (d) FOM and (e) OM images taken after irradiation with green light for $60 \mathrm{~min}$. The white inset line profiles in $(b, c, d)$ indicate changes in the relative fluorescence intensities corresponding to the white arrow lines.

agglomerate together to generate macroscale fiber wads. As clearly seen in the FOM image (Fig. 2b), compared with isolated nanofibers, highly entangled fiber wads displayed stronger red fluorescence, confirming AIE/AIEE characteristics. On the other hand, these dried fibrous structures did not show fluorescence when excited with UV or blue light from a mercury lamp.

We next investigated the light-responsiveness of $3 \mathrm{BrAz}$ nanostructures. The inset profiles in Fig. 2b$2 \mathrm{~d}$ exhibit the variations in the relative fluorescence intensities corresponding to the white lines in the FOM images. The fluorescence intensity of $3 \mathrm{BrAz}$
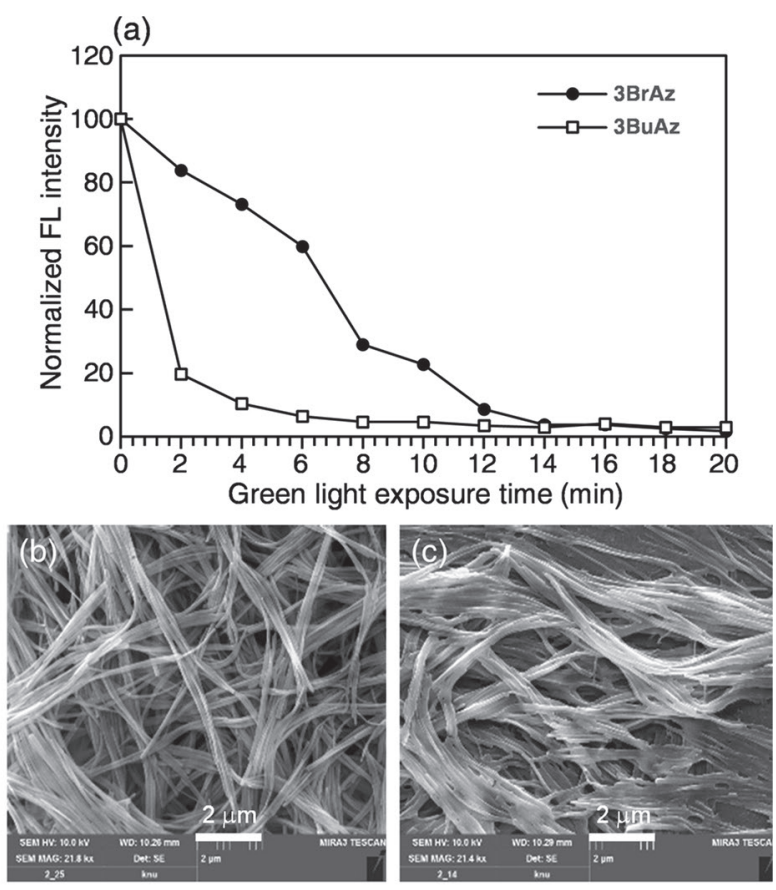

Fig. 3. (a) Changes in relative fluorescence intensities of $3 \mathrm{BrAz}$ nanofibers and $3 \mathrm{BuAz}$ spheres as a function of green light irradiation time. SEM images of $3 \mathrm{BrAz}$ nanofibers (b) before and (c) after exposure to green light for $60 \mathrm{~min}$. nanofiber was reduced by approximately $40 \%$ after irradiation with light for 6 min (Figs. 2c and 3a). Additional irradiation with green light for $10 \mathrm{~min}$ led to significant decreases in the red fluorescence intensity, and consequently the fluorescence almost disappeared (Fig. 2d). The OM image taken after green light irradiation for $60 \mathrm{~min}$ demonstrates that the relatively isolated red fibers turned orangebrown, while the largely entangled fiber wads retained their original brown color (Fig. 2e). Such slow decoloration and fluorescence decrease seems to be caused not by light-induced isomerization of 3BrAz, but by prolonged light irradiation.

To obtain more information about the morphological changes during green light irradiation, we conducted SEM measurements. Compared with the as-prepared fibrous structures (Fig. 3b), the largely entangled fibers kept their onedimensional shapes almost intact even after green light irradiation for $60 \mathrm{~min}$ (Fig. 3c). But, the relatively isolated fibers looked somewhat flat. The slight morphological changes seem to be associated with the prolonged light irradiation which might disturb fast thermal back isomerization and might raise the temperature of the sample to some extent.

For comparison, red fluorescence from $3 \mathrm{BuAz}$ spheres decreased drastically after irradiation with green light for 1-2 min and fully vanished after irradiation for 4 min (Figs. 3a and 4). The resulting liquefaction of spherical objects was observed clearly. As a consequence, the relatively fast light response is attributed to fast and sufficient photoisomerization of $3 \mathrm{BuAz}$ molecule and slow thermal back isomerization $[17,18]$.

\section{Conclusion}

We have presented light-induced fluorescence, color and morphological changes in fluorescent self-assembled aggregates composed of 


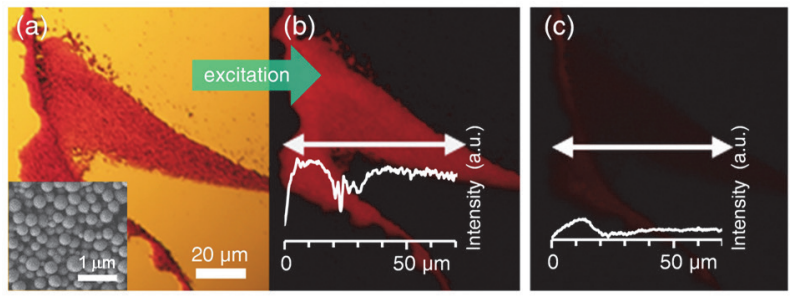

Fig. 4. (a) OM, SEM (inset) and (b) FOM images of 3BuAz spheres. (c) FOM image taken after irradiation with green light for $2 \mathrm{~min}$. The inset line profiles in (b and c) indicate changes in the relative fluorescence intensities corresponding to the white arrow lines.

azobenzene-containing chromophores. Successive visible light irradiation of nanofibers generated from $3 \mathrm{BrAz}$ chromophore showing a very small amount of photoisomerization resulted in slow decoloration and fluorescence decrease, but the morphology was hardly affected by light. These results support that light-induced morphological changes seem to be mainly derived from large changes in the molecular structure of the constituent molecules. Our investigations suggest that an important key to realizing fast and efficient light response depends on molecular design strategies for photochromic compounds showing sufficient photoisomerization.

\section{Acknowledgement}

This work was supported by a research grant from Kongju National University in 2018.

\section{References}

1. 1. A. P. de Silva, H. Q. N. Gunaratne, T. Gunnlaugsson, A. J. M. Huxley, C. P. McCoy, J. T. Rademacher, and T. E. Rice, Chem. Rev., 97 (1997) 1515.

2. Y. Sagara and T. Kato, Nat. Chem., 1 (2009) 605.

3. Y. Hong, J. Lam, and B. Z. Tang, Chem. Soc. Rev., 40 (2011) 5361.
4. B.-K. An, J. Gierschner, and S. Y. Park, Acc. Chem. Res., 45 (2012) 544.

5. Z. Chi, X. Zhang, B. Xu, X. Zhou, C. Ma, Y. Zhang, S. Liu, and J. Xu, Chem. Soc. Rev., 41 (2012) 3878 .

6. S. I. Stupp and L. C. Palmer, Chem. Mater., 26 (2014) 507.

7. T. L. Mako, J. M. Racicot, and M. Levine, Chem. Rev., 119 (2019) 322.

8. J.-M. Lehn, "Supramolecular Chemistry", VCH Press, New York (1995).

9. L. F. Lindoy and I. M. Atkinson, "Self-assembly in Supramolecular Systems", Ed., J. F. Stoddart, Royal Society of Chemistry, Cambridge, UK (2000).

10. G. M. Whitesides, J. P. Mathias, and C. T. Seto, Science, 254 (1991) 1312.

11. "Molecular Switches", Eds., B. L. Feringa and W. R. Browne, Wiley-VCH, Weinheim, Germany (2011).

12. T. Kobayashi, Y. Kitamoto, Y. Hirai, T. Kajitani, T. Seki, and S. Yagai, Commun. Chem., 1 (2018) 58.

13. I. Abe, M. Hara, T. Seki, S. J. Cho, M. Shimizu, K. Matsuura, H. K. Cheong, J. Y. Kim, J. Oh, J. Jung, and M. Han, J. Mater. Chem. C, 7 (2019) 2276.

14. M. Han, S. J. Cho, Y. Norikane, M. Shimizu, A. Kimura, T. Tamagawa, and T. Seki, Chem. Commun., 50 (2014) 15815.

15. "Photoreactive Organic Thin Films", Eds., Z. Sekkat and W. Knoll, Academic Press, Elsevier Science, USA (2002).

16. H. Rau, "Photochromism: Molecules and Systems", Eds., H. Dürr and H. Bouas-Laurent, Elsevier, Amsterdam (1990).

17. Y. Norikane, Y. Hirai, and M. Yoshida, Chem. Commun., 47 (2011) 1770.

18. I. Abe and M. Han, New J. Chem., 43 (2019) 19014. 